\title{
Prevalence of vertebral fractures on chest radiographs of elderly African American and Caucasian women
}

\author{
D. Lansdown $\cdot$ B. Bennet $\cdot$ S. Thiel $\cdot$ O. Ahmed $\cdot$ \\ L. Dixon - T. J. Vokes
}

Received: 30 July 2010 /Accepted: 24 September 2010/Published online: 9 November 2010

(C) The Author(s) 2010. This article is published with open access at Springerlink.com.

\begin{abstract}
Summary The prevalence of vertebral fractures on routine chest radiographs of elderly Caucasian women was only 1.3 times higher than in African American (AA) women, a difference considerably smaller than reported in population studies. AAs with medical problems may
\end{abstract}

Presented in part at the 2007 Endocrine Society Annual Meeting and the 2007 American Society for Bone and Mineral Research Annual Meeting

D. Lansdown • B. Bennet $\cdot$ T. J. Vokes $(\bowtie)$

Section of Endocrinology, Department of Medicine,

University of Chicago,

5841 S. Maryland, MC1027,

Chicago, IL 60637, USA

e-mail: tvokes@uchicago.edu

S. Thiel $\cdot$ O. Ahmed $\cdot$ L. Dixon

Department of Radiology, University of Chicago,

Chicago, IL, USA

Present Address:

D. Lansdown

Department of Orthopedic Surgery, University of California,

San Francisco,

San Francisco, CA, USA

Present Address:

B. Bennet

Dreyer Medical Clinic,

Aurora, IL, USA

Present Address:

S. Thiel

Suburban Imaging,

Minneapolis, MN, USA have higher risk of vertebral fractures than previously suspected.

Introduction Earlier studies noted a 1.9- to 3.7-fold higher prevalence of vertebral fractures in Caucasian (CA) compared to African American (AA) women. These studies, however, may have suffered from selection bias. We reported that among women referred for bone density testing, the prevalence of vertebral fractures in AA was the same as in CA women. Suspecting that the latter might have been due to a referral bias, we examined the racial difference in the prevalence of vertebra fractures on chest radiographs of patients seeking general medical care, not selected for osteoporosis.

Methods Consecutive chest radiographs $(N=1,200)$ of women over age 60 were evaluated using Genant's semiquantitative method. Patients' race and the presence of diseases or medications associated with increased fracture risk were ascertained from the electronic medical records.

Results Among 1,011 women (76\% AA) with usable radiographs, $11 \%$ had moderate or severe vertebral fractures. The prevalence of vertebral fractures was $10.3 \%$ in $773 \mathrm{AA}$ and $13 \%$ in 238 CA women ( $p=0.248$ for difference between races). The lack of difference persisted after controlling for age, smoking, use of glucocorticoids, or presence of cancer, rheumatoid arthritis, organ transplantation, and end-stage renal disease. Among all subjects, CA women were more likely to be diagnosed and treated for osteoporosis $(p<0.001)$. Conclusion Among subjects seeking medical care, the difference in the prevalence of vertebral fractures between $\mathrm{AA}$ and $\mathrm{CA}$ women is smaller than previously suspected. Greater attention to the detection of vertebral fractures and the management of osteoporosis is warranted in AA women with medical problems.

Keywords African American - Caucasian - Osteoporosis . Race $\cdot$ Racial disparity $\cdot$ Vertebral fractures 


\section{Introduction}

Vertebral fractures are important to detect because they are associated with significant morbidity, mortality, and reduced quality of life $[1,2]$ and because they strongly predict future fractures [3-6] and are considered diagnostic of osteoporosis. Clinical vertebral fractures (i.e., those that are clinically recognized) comprise only one third of all fractures found on radiographs [7-9]. However, radiographic vertebral fractures are also indicative of osteoporosis and predictive of future fracture risk. Therefore, spine imaging is necessary to assess the true prevalence of vertebral fractures in a given population. Knowing the prevalence of vertebral fractures in different populations aids the quantification of the osteoporotic burden and facilitates better management of this condition.

It is generally accepted that compared to Caucasian Americans (CA), African Americans (AA) have a lower risk of osteoporotic fractures. Consequently, AA are less likely to undergo appropriate diagnostic procedures or receive therapies for osteoporosis even when they present with fractures or use medications that cause bone loss [1012]. In 1997, Jacobsen et al. analyzed Medicare discharge diagnoses and reported higher rates of clinical vertebral fractures in CA than in AA women (17.1 vs. 3.7 per 10,000 per year) [13]. The authors acknowledged that these results might have been partly due to a bias if physicians suspected vertebral fractures and performed necessary imaging in CA patients but not in AA patients presenting with back pain. A different kind of bias may affect population studies of osteoporosis, most of which focused on CA women with under-representation of AA women. Two such studies have examined vertebral fractures. The National Osteoporosis Risk Assessment reported numerically higher 1-year incidence of clinical vertebral fractures in CA than in AA women $(0.185 \%$ vs. $0.12 \%)$, but the difference was not statistically significant [14]. In the study of osteoporotic fractures, the prevalence of vertebral fractures was higher in CA than in AA women (19.1\% vs. 10.6\%) [15]. However, population studies tend to enroll relatively healthier subjects in general, and this may be particularly true for AA participants. As a result, the difference in the health level between the study subjects and that of the general population may be exaggerated for AA subjects.

We have observed that among women referred for bone densitometry at a university hospital with a large percentage of AA patients, the prevalence of vertebral fractures was similar in AA and CA women [16]. This may be due to a referral bias if AA women are referred for bone mineral density when their treating physician has high suspicion for fractures while CA women are referred for screening purposes. Alternatively, the true prevalence of vertebral fractures in AA may be underestimated in the above- mentioned population studies, which may have preferentially recruited healthier subjects. Chest radiographs have previously been utilized to examine the under-reporting of vertebral fractures $[9,17,18]$ and can be used to estimate disease prevalence in subjects not selected for osteoporosis screening. To obtain an unbiased estimate of racial differences in vertebral fracture burden in subjects seeking medical care, we examined the prevalence of vertebral fractures on lateral chest radiographs obtained for routine clinical purposes.

\section{Methods}

All consecutive chest radiographs from women over the age of 60 were collected for the calendar years of 2005 and 2006 and sorted by medical record number (MRN). The first 600 MRNs from 2005 and the first 600 MRNs from 2006 were included in the study. Electronic medical records were used to obtain clinical information for each patient whose radiograph was included in the analysis. The study was approved by the University of Chicago's Institutional Review Board.

\section{Evaluation of radiographs}

The chest radiographs were available in digital form and accessed using Philips iSite v. 3.3.2 (Stentor). Evaluation of radiographs was done without knowledge of the race or other clinical characteristics of the patients. Vertebral fractures were classified using Genant's semi-quantitative scale [19], which defines a grade 1 fracture as a loss of vertebral height of 20 $25 \%$, grade 2 as a loss of $26-40 \%$, and grade 3 as a loss of greater than $40 \%$. A spinal deformity index (SDI) was calculated for each patient as the sum of the fracture grades of all vertebrae from that patient [20]. Patients with an SDI of at least 2 were classified as having a fracture.

\section{Information from the medical records}

Electronic medical records were used to ascertain the race of the patient, when available, as well as the presence of conditions or use of medications that may be associated with an increased risk of fractures including: a history of cancer, use of systemic (but not inhaled) glucocorticoids, rheumatoid arthritis, organ transplantation, end-stage renal disease (ESRD), and cigarette smoking. It was also noted whether the patient had a bone mineral density (BMD) result available in the electronic record, and if so, whether there was osteoporosis by BMD defined as a T-score of $\leq-2.5$ at the lumbar spine, femoral neck, or total hip. A diagnosis of osteoporosis by medical record was present if the diagnosis of osteoporosis was recorded in the physicians' notes. Treatment of osteoporosis was present if the patient was receiving 
calcium, with or without vitamin $\mathrm{D}$, or pharmacologic therapy for osteoporosis (bisphosphonates, estrogen, raloxifene, teriparatide, or calcitonin). It should be noted that at the time of the study, the electronic medical record contained the progress notes only for some clinics, and the ascertainment of the medication use and medical problems present may thus be incomplete.

\section{Statistical analysis}

Statistical analyses were performed using STATA 10 (StataCorp, College Station, TX) software. Differences between AA and CA patients were examined using a $t$ test for continuous and chi-squared test for categorical variables. Logistic regressions were used to determine whether the observed difference in the prevalence of vertebral fractures between the $\mathrm{AA}$ and $\mathrm{CA}$ women could be explained by medical conditions associated with osteoporosis (see above). In these logistic regression analyses, presence of vertebral fractures (yes or no) was a binary outcome while race (AA or CA) and age were fixed predictors in all models. The conditions associated with osteoporosis were then added one at a time to the model as covariates. In addition, interaction terms with race were generated for each of these covariates and added into the model along with the respective covariate, race, and age.

\section{Results}

After eliminating duplicate exams from the same patients, uninterpretable images, women who were not AA or CA, or patients without a race specified, there were 1,011 subjects left for analysis. Their clinical characteristics are shown in Table 1. The two racial groups did not differ in age, prevalence of rheumatoid arthritis, previous organ transplantation, or systemic glucocorticoid usage. CA women were more likely to have a history of cancer, but they had a lower prevalence of end-stage renal disease and smoking. A higher percentage of AA received their primary care at the University of Chicago Medical Center.

There was no statistically significant difference between the races in the vertebral fracture prevalence (Table 1 and Fig. 1) or vertebral fracture burden measured by the spinal deformity index (SDI of $3.2 \pm 2.3$ in AA vs. $3.4 \pm 2.0$ in CA women with vertebral fractures, $p=0.66$ ). When the data were stratified according to decade of age (60-69, 70-79, and 80 years and older), the fracture prevalence was significantly higher in CA than in AA aged 60-70 years but not in the older age strata (Fig. 1). The prevalence of vertebral fractures increased with age in AA but not in CA women in whom the prevalence of vertebral fractures was relatively higher in 60-70 years old compared to the older women (Fig. 1). The proportion of women who had the diagnosis of cancer decreased with age in CA women, although this difference was not statistically significant $(p=$ 0.3 ). The lack of age-related increase in the prevalence of vertebral fractures was observed in CA women with as well as those without cancer.

The conditions from Table 1 that may influence vertebral fracture risk were examined separately in patients with vertebral fractures. Although there were differences in the frequency of these conditions in the whole population, these differences did not reach statistical significance when the analysis was restricted to women with vertebral fractures (data not shown).

We used logistic regression to determine whether the lack of a significant racial difference in the prevalence of vertebral fractures could be explained by a differential burden of conditions associated with osteoporosis. In these logistic regression models, the presence of vertebral fracture(s) was an outcome variable, race and age were fixed predictors, and each of the clinical characteristics from Table 1 was added individually to the model as a covariate. There was no significant effect of any of the clinical conditions and no significant interaction of these clinical variables with race. Furthermore, the point estimates for race (coefficients) in the regression models
Table 1 Clinical characteristics of 1,011 women whose chest radiographs were used in analysis

The results are given as mean \pm standard deviation for continuous variables and number (percent) for categorical variables. $p$ values are from $t$ test for continuous and chisquare test for categorical variables $E S R D$ end-stage renal disease, $P C P$ primary care physician

\begin{tabular}{lllc}
\hline Clinical characteristic & Caucasian $(N=238)$ & African American $(N=773)$ & $p$ value \\
\hline Age (years) & $74.9 \pm 8.5$ & $74.5 \pm 8.7$ & 0.50 \\
Vertebral fracture & $31(13.0 \%)$ & $80(10.4 \%)$ & 0.26 \\
Cancer & $85(35.7 \%)$ & $147(19.0 \%)$ & $<0.001$ \\
Rheumatoid arthritis & $6(2.5 \%)$ & $20(2.6 \%)$ & 0.96 \\
ESRD & $3(1.3 \%)$ & $43(5.6 \%)$ & 0.005 \\
Transplant & $5(2.1 \%)$ & $9(1.2 \%)$ & 0.28 \\
Glucocorticoids & $20(8.4 \%)$ & $44(5.7 \%)$ & 0.13 \\
Smoking & $40(18.5 \%)$ & $223(28.9 \%)$ & 0.002 \\
PCP at Univ. of Chicago & $117(49.2 \%)$ & $522(67.5 \%)$ & $<0.001$ \\
\hline
\end{tabular}


Fig. 1 Prevalence of vertebral fractures according to age in Caucasian and African American women. The absolute numbers of patients with fractures are shown above each bar graph together with the number of patients in respective age/race strata

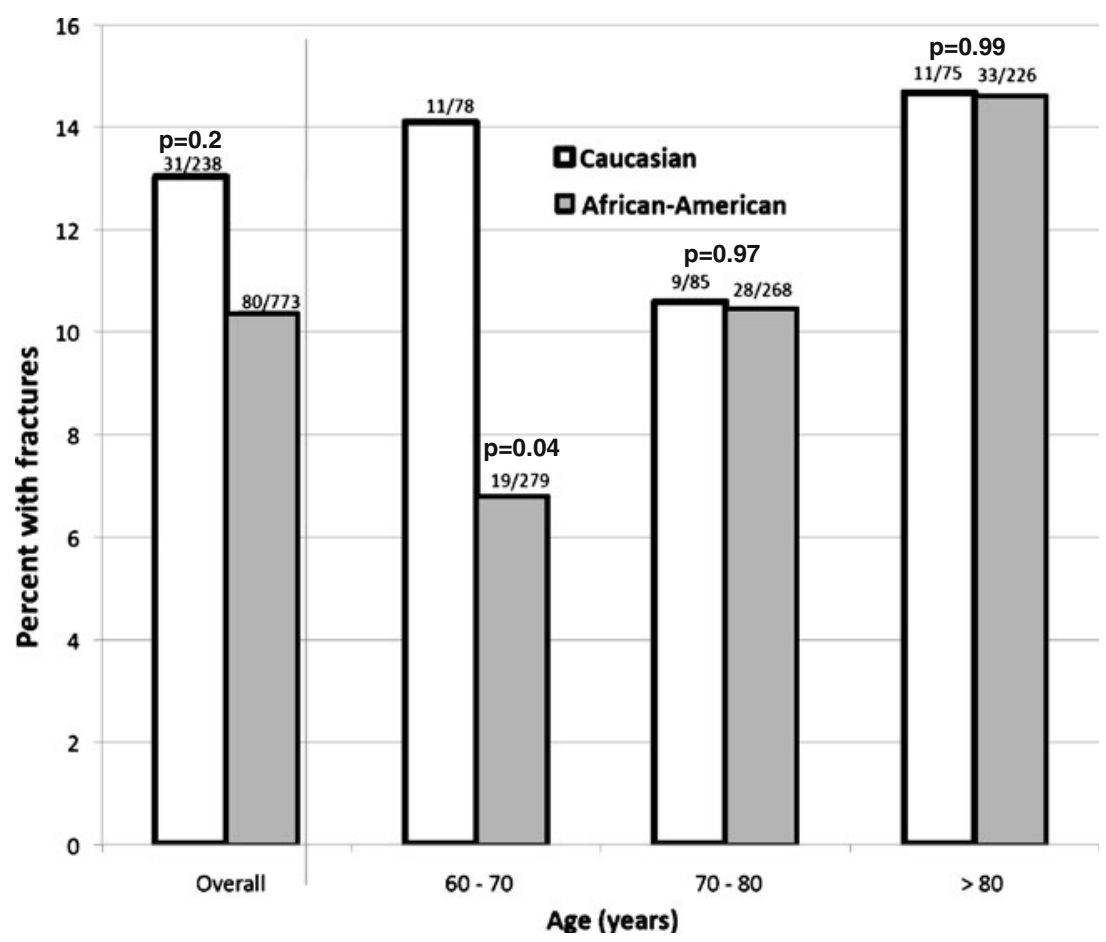

were not significantly affected by adding any of the clinical conditions.

While cancer was more prevalent in CA women, the racial differences in vertebral fractures were similar in women with and without cancer (Fig. 2a). Although among all subjects, smoking was more common in the AA group, the rates of smoking did not differ between $\mathrm{AA}$ and CA women with vertebral fractures. When stratifying the analysis by smoking, the racial difference in vertebral fracture prevalence was more pronounced in smokers, although the difference did not reach statistical significance (Fig. 2b). ESRD was more common among AA women. However, the difference in the prevalence of vertebral fractures between the two racial groups was similar in 965 subjects without ESRD ( $10 \%$ in AA vs. $13.2 \%$ in CA, $p=$ 0.2 ) and in the whole population. The racial difference in vertebral fracture prevalence was more pronounced in women with history of systemic glucocorticoid use than in those without (Fig. 2c), although this was not statistically significant. The prevalence of vertebral fractures did not differ between subjects who had and those who did not have primary care physician at the University of Chicago (Fig. 2d).

Less than half of the subjects had results of BMD testing in the medical record with no racial difference in the percentage of subjects tested (Table 2). CA women were more likely to have a BMD diagnosis of osteoporosis defined as T-scores $\leq-2.5$ at either the lumbar spine or the proximal femur. CA women were also more likely to have a diagnosis of osteoporosis recorded in the medical record and to receive treatment for osteoporosis (Table 2). Similar trends were observed in women with vertebral fractures (Table 3). Higher proportions of CA women received pharmacologic treatment for osteoporosis $(p=0.02)$.

Only $18 \%$ of patients with vertebral fractures found on chest radiographs in this study had vertebral fractures mentioned in the radiology report, with no significant difference between the races.

\section{Discussion}

We have previously observed that among patients referred for bone density testing at the University of Chicago, the prevalence of vertebral fractures was similar in AA and CA women [16]. In contrast, population studies reported that the prevalence of vertebral fractures in CA women was 1.9- to 2.3-fold higher [14, 15]. Since population studies often recruit subjects who are relatively healthier than the general population and certainly healthier than subjects who are seeking medical care, we wanted to obtain an unbiased estimate of true racial difference in the prevalence of vertebral fractures in patients cared for at a medical facility. To this end, we examined consecutive chest radiographs of elderly AA and CA women and found that the racial difference in vertebral fracture prevalence was considerably smaller (only 1.3-fold higher in CA women) and not statistically significant. We then investigated whether this unexpected observation could be explained by differences in medical conditions which lead 
Fig. 2 Prevalence of vertebral fractures in Caucasian (open bars) and African American women (shaded bars) according to presence of cancer (a), smoking (b), use of glucocorticoids ( $\mathrm{GC}$ - graph $\mathbf{C}$ ), or having primary care physician (PCP) at the University of Chicago (d)
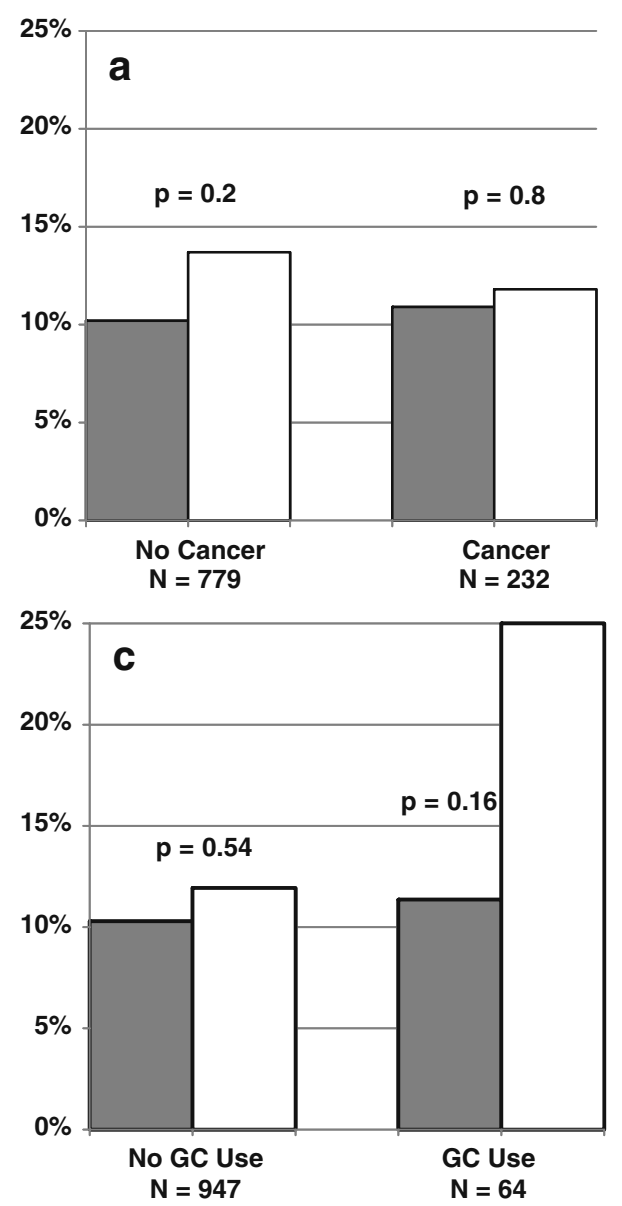

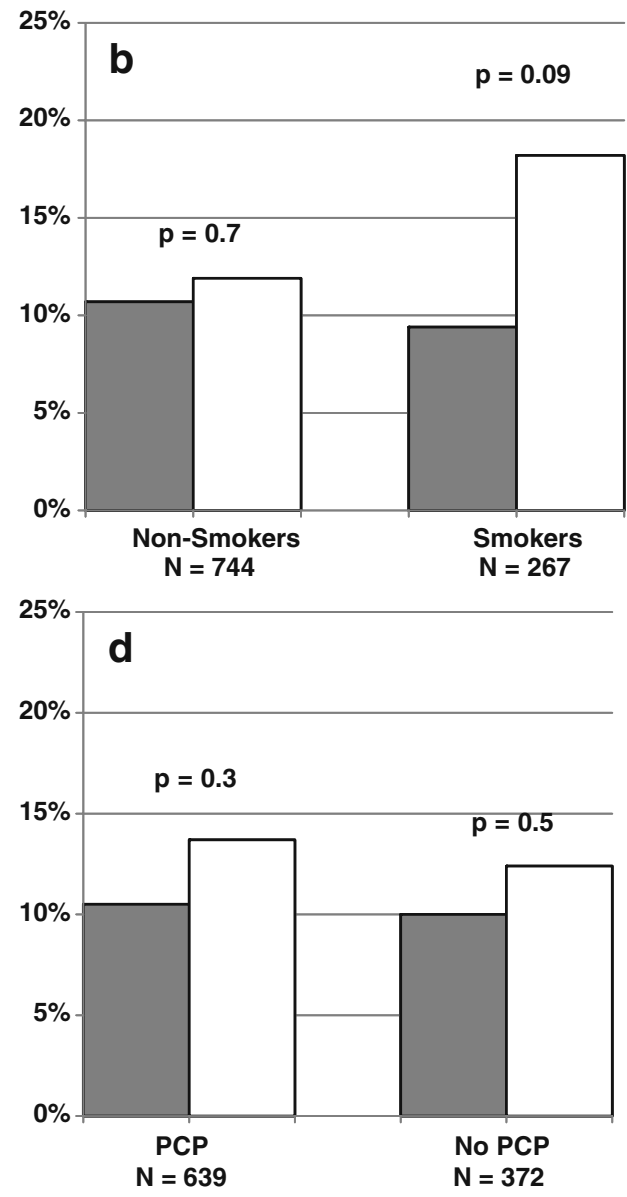

to osteoporosis and vertebral fractures. Our results suggest that this is not the case.

The two races were similar in age, which is a known strong predictor of vertebral fractures. When medical conditions that may be associated with fractures (Table 1) were added as covariates to regression analyses with vertebral fractures as outcome, and race and age as fixed predictors, the point estimates (coefficients) for race did not change. None of the medical conditions examined had a significant effect in the regression models or significant interaction with race. Cancer was present in a higher proportion of CA women. However, that should result in a greater, rather than smaller, difference in the vertebral fracture prevalence between $\mathrm{CA}$ and $\mathrm{AA}$ women, assuming that some of the fractures are due to malignant causes or to osteoporosis resulting from treatment for malignancy. The
AA group had higher prevalence of ESRD, but the racial differences in the vertebral fracture prevalence were similar in patients without ESRD and in the whole study sample. We also observed higher prevalence of smoking in the AA subjects. Interestingly, we found greater (albeit not statistically significant) racial difference in the vertebral fracture prevalence among smokers than non-smokers (Fig. 2b). It is possible that this was due to a difference in body weight (lower weight in CA as compared to AA smokers) which was not available in our study. We found grater racial difference in vertebral fracture prevalence (again not statistically significant) in women with history of glucocorticoid use (Fig. 2c). However, we did not have an accurate estimate of the glucocorticoid dose, which makes any conclusion regarding the racial differences in its effect unreliable.
Table 2 Osteoporosis (OP) diagnosis and management-all subjects

\footnotetext{
${ }^{\text {a }}$ Among the $110 \mathrm{CA}$ and $317 \mathrm{AA}$
} women who had BMD testing

\begin{tabular}{lccr}
\hline & Caucasian $(N=238)$ & African American $(N=773)$ & $p$ value \\
\hline BMD in medical record & $110(46.2 \%)$ & $317(41.0 \%)$ & 0.155 \\
OP on BMD & $42(38.2 \%)$ & $71(22.4 \%)$ & 0.001 \\
OP in medical record & $44(18.5 \%)$ & $64(8.3)$ & $<0.001$ \\
Calcium \pm vitamin D & $72(30.3 \%)$ & $104(13.5 \%)$ & $<0.001$ \\
Pharmacologic therapy & $55(23.1 \%)$ & $66(8.5 \%)$ & $<0.001$ \\
\hline
\end{tabular}


Table 3 Osteoporosis (OP) diagnosis and management in women with vertebral fractures

${ }^{a}$ Among the $13 \mathrm{CA}$ and $38 \mathrm{AA}$ women with fractures who had BMD testing

\begin{tabular}{lccc}
\hline & Caucasian $(N=31)$ & African American $(N=80)$ & $p$ value \\
\hline BMD in medical record & $13(41.9 \%)$ & $38(47.5 \%)$ & 0.598 \\
OP on BMD & $8(61.5 \%)$ & $13(34.2 \%)$ & 0.084 \\
OP in medical record & $8(25.8 \%)$ & $13(16.3 \%)$ & 0.249 \\
Calcium \pm vitamin D & $8(25.8 \%)$ & $15(18.8 \%)$ & 0.411 \\
Pharmacologic therapy & $12(38.7)$ & $14(17.5 \%)$ & 0.018 \\
\hline
\end{tabular}

We also entertained the possibility that our observation may be due to heterogeneity of our study sample, which included both patients who received their primary care at our institution and those who were referred for tertiary care. We found similar racial differences in vertebral fracture prevalence among patients who were and those who were not receiving primary care at the University of Chicago (Fig. 2d). It appears, therefore, that our observation of smaller than expected difference in the vertebral fracture prevalence between AA and CA women are not explained by easily identifiable differences in health status.

Age is one of the most important risk factors for the development of osteoporotic vertebral fractures. Therefore, we stratified the analysis by decade and found a racial difference only for the youngest age strata (60-70 years). As expected, in AA, the prevalence of vertebral fractures increased with age (Fig. 1). In contrast, the fracture prevalence in the CA group decreased between the sixth and seventh decades before increasing again. A greater proportion of younger CA women had the diagnosis of cancer, but this does not fully explain our data as a similar pattern was observed in women with and without cancer. The reason for the unusual age distribution of vertebral fractures in our CA subjects remains unclear and may be due to a relatively small sample size of CA women. Based on our data, it is possible that CA women start having vertebral fractures at an earlier age (60-70 years old), while the racial difference in vertebral fracture rates becomes smaller or non-existent with more advanced age (over 70 years of age). The cross-sectional nature of our study precludes any firm conclusions regarding this question.

The reason for a relatively higher than expected prevalence of vertebral fractures in AA relative to $\mathrm{CA}$ women in our study is thus not explained by any of the risk factors we could assess through the medical record review. We hypothesize that the racial differences in fracture rates observed in healthier participants in population studies are diminished in patients seeking medical care, who are probably sicker. The mechanism by which "being sick" increases fracture risk is currently unclear but may involve low physical activity, hypogonadism, effect of other metabolic diseases, or vitamin D deficiency. Further studies are needed to explore these possibilities and to develop therapeutic approaches to correct them.
A similar percentage of $\mathrm{AA}$ and $\mathrm{CA}$ subjects in our study had BMD documented in their medical record, which suggests that there was no major racial disparity in screening for osteoporosis. Nevertheless, Caucasian women were more likely to have a diagnosis of osteoporosis in their medical records, and they were also more likely to receive treatment for osteoporosis. Among women with vertebral fractures, the racial differences reached statistical significance only for treatment but not for diagnosis of osteoporosis (Table 3). A majority of women with vertebral fractures identified in this study were not diagnosed with osteoporosis: only $25.8 \%$ of CA and $16.3 \%$ of AA women with vertebral fractures had osteoporosis mentioned in their medical record. The rates of treatment for osteoporosis were low, particularly for AA women (Table 3).

The fracture prevalence in our study population of $11 \%$ is slightly lower than the $14-16 \%$ prevalence reported in other studies of chest radiographs [9, 17]. The lower prevalence in our study may be due to the fact that we included both inpatient and outpatient exams while the previous studies utilized chest radiographs of hospitalized patients [9] or patients who presented to the emergency room [17]. Both of these patient groups may be relatively sicker than our study population, which included potentially healthier outpatients.

There are some limitations to our study. First, because we examined chest radiographs, we could not detect most of the fractures in the lumbar spine. However, this is true for both races and not likely to affect the comparison. A second limitation is that we assessed the health status using electronic medical records, which may be incomplete for some of the patients, but this should affect the two races equally. We also relied on medical records to determine the race of a patient. Again, any errors should be randomly distributed between the two groups.

This study also has significant strengths. It is the first study to date to examine vertebral fractures in a population with a large proportion of African Americans, the population group in which osteoporosis is more likely to be underrecognized [10, 12]. In addition, we included a thorough review of medical records, which allowed us to examine whether our observations may be due to racial differences in health status. 
The results of this study may have significant implications for the diagnosis and treatment of osteoporosis in the AA community. AA currently receive fewer diagnostic, therapeutic, and preventative measures for osteoporosis because it is assumed that they are less affected by this disease [12]. While this may be true for a healthy population, our results suggest that among those seeking medical care, AA are affected by osteoporosis at rates that are much closer to those of CA subjects. This is consistent with a study of a COPD cohort, which reports similar rates of vertebral fractures in AA and CA patients [21]. Based on these findings, it may be prudent to increase attention to osteoporosis and vertebral fractures in AA subjects with medical problems.

Acknowledgement Grant support: K23 AR048205-01A1 from the National Institute of Health

\section{Conflicts of interest None.}

Open Access This article is distributed under the terms of the Creative Commons Attribution Noncommercial License which permits any noncommercial use, distribution, and reproduction in any medium, provided the original author(s) and source are credited.

\section{References}

1. Burger $\mathrm{H}$ et al (1997) Vertebral deformities and functional impairment in men and women. J Bone Miner Res 12(1):152-157

2. Cockerill W et al (2004) Health-related quality of life and radiographic vertebral fracture. Osteoporos Int 15(2):113-119

3. Cauley JA et al (2007) Long-term risk of incident vertebral fractures. JAMA 298(23):2761-2767

4. Delmas PD et al (2003) Severity of prevalent vertebral fractures and the risk of subsequent vertebral and nonvertebral fractures: results from the MORE trial. Bone 33(4):522-532

5. Lindsay R et al (2001) Risk of new vertebral fracture in the year following a fracture. Jama 285(3):320-323
6. Melton LJ 3 rd et al (1999) Vertebral fractures predict subsequent fractures. Osteoporos Int 10(3):214-221

7. Cooper C, O'Neill T, Silman A (1993) The epidemiology of vertebral fractures. European Vertebral Osteoporosis Study Group. Bone 14(Suppl 1):S89-S97

8. Fink HA et al (2005) What proportion of incident radiographic vertebral deformities is clinically diagnosed and vice versa? J Bone Miner Res 20(7):1216-1222

9. Gehlbach SH et al (2000) Recognition of vertebral fracture in a clinical setting. Osteoporos Int 11(7):577-582

10. Curtis JR et al (2005) Longitudinal patterns in the prevention of osteoporosis in glucocorticoid-treated patients. Arthritis Rheum 52(8):2485-2494

11. Cheng $\mathrm{H}$ et al (2009) Estimated prevalence and patterns of presumed osteoporosis among older Americans based on Medicare data. Osteoporos Int 20(9):1507-1515

12. Curtis JR et al (2009) Population-based fracture risk assessment and osteoporosis treatment disparities by race and gender. J Gen Intern Med 24(8):956-962

13. Jacobsen SJ et al (1992) Hospitalization with vertebral fracture among the aged: a national population-based study, 1986-1989. Epidemiology 3(6):515-518

14. Barrett-Connor E et al (2005) Osteoporosis and fracture risk in women of different ethnic groups. J Bone Miner Res 20(2):185194

15. Cauley JA et al (2008) Prevalent vertebral fractures in black women and white women. J Bone Miner Res 23(9):14581467

16. Vokes TJ et al (2007) Risk factors for prevalent vertebral fractures in black and white female densitometry patients. J Clin Densitom 10(1):1-9

17. Majumdar SR et al (2005) Incidental vertebral fractures discovered with chest radiography in the emergency department: prevalence, recognition, and osteoporosis management in a cohort of elderly patients. Arch Intern Med 165(8):905-909

18. Mui LW et al (2003) Evaluation of vertebral fractures on lateral chest radiographs of inner-city postmenopausal women. Calcif Tissue Int 73(6):550-554

19. Genant HK et al (1993) Vertebral fracture assessment using a semiquantitative technique. J Bone Miner Res 8(9):1137-1148

20. Crans GG, Genant HK, Krege JH (2005) Prognostic utility of a semiquantitative spinal deformity index. Bone 37(2):175-179

21. Li L et al (2008) African Americans and men with severe COPD have a high prevalence of osteoporosis. COPD 5(5):291-297 